PHYSICAL REVIEW D 90, 062003 (2014)

\title{
Gravitational lensing effects on parameter estimation in gravitational wave detection with advanced detectors
}

\author{
Zhoujian Cao* \\ Institute of Applied Mathematics, Academy of Mathematics and Systems Science, \\ Chinese Academy of Sciences, Beijing 100190, China \\ Li-Fang $\mathrm{Li}^{\dagger}$ \\ State Key Laboratory of Space Weather, Center for Space Science and Applied Research, \\ Chinese Academy of Sciences, Beijing 100190, China \\ Yan Wang \\ Albert Einstein Institute, Callinstrasse 38, D-30167 Hanover, Germany and QUEST Centre for Quantum \\ Engineering and Space-Time Research, Leibniz University of Hanover, D-30167 Hanover, Germany \\ (Received 19 December 2013; revised manuscript received 17 June 2014; published 4 September 2014)
}

The gravitational lensing effect is important to the detection of electromagnetic signals in astrophysics. The gravitational wave lensing effect has also been found significant to gravitational wave detection in the past decade. Recent analysis shows that the lensing events for advanced detectors could be quite plausible. The black holes in our Milky Way Galaxy may play the role of lens objects. These facts motivate us to study the lensing effects on gravitational wave signals for advanced detectors. Taking advanced LIGO and Einstein Telescope for examples, we investigate the lensing effects on the parameter extraction of gravitational wave signals. Using the Markov chain Monte Carlo simulation together with matched filtering methods, we find that the lensing effect for a lens object with small mass is negligible. But when the mass of the lens object increases to larger than $1000 M_{\odot}$ the lensing effect becomes important. Using the template without lensing corrections would result in loss of signal detections. In contrast if we consider templates with lensing effects, the lensed signal may provide much information about the lens black hole. These facts may give us a new way to determine the parameters of the lensing object. For example, this kind of signal may also help us estimate the mass and the distance of the supermassive black hole hosted at the center of our Galaxy.

DOI: 10.1103/PhysRevD.90.062003

PACS numbers: 04.80.Nn, 04.25.Nx

\section{INTRODUCTION}

Weak lensing is of capital importance in electromagnetic observations. Due to the similarity between gravity and electromagnetism, people have done analogous research with optics approximation in the gravitational lensing of gravitational waves in the 1970s [1,2]. Because the detectable gravitational wave event rates are small, the lensed gravitational wave event rate is low. There have been few motivations for a long time to consider the lensing effect on gravitational waves. Until recently, the authors in [3,4] realized that the gravitational lensing effect on gravitational waves should be described by wave dynamics instead of optics approximation. The features of wave dynamics make the fraction of lensed events larger than that optics approximation has ever predicted. This fact motivated many authors to study lensing effects on gravitational waves in the past decade [3-16]. As noted in [4], the lensing effect is very important for the space-based gravitational wave detector

\footnotetext{
zzjcao@amt.ac.cn

†ilf@itp.ac.cn

\#an.wang@aei.mpg.de
}

LISA [17]. Even for the coming generations of ground-based gravitational wave detectors such as Advanced LIGO and Einstein telescope (ET), lensing effects are also nonnegligible. This is because the detectable binary sources for these detectors may reach redshifts $z \geq 1$ [18]. The authors in [19] found that the gravitational lensing effects may be significant to ET. They only considered neutron star-neutron star (NS-NS) inspiral as a gravitational wave source and event rate for gravitational wave lensing roughly tens per year and one per decade in the most optimistic case and in the most pessimistic case respectively.

The direct detection of gravitational wave signals is important for fundamental science. Yet extracting information from the gravitational wave signal is much more useful, which is the foundation of the gravitational wave astronomy. The accuracy of the parameter estimation in gravitational wave detection will affect many aspects of science study and will affect the development of gravitational wave astronomy. For example, if we can extract the distance and the angular position of the gravitational wave source accurately, this information may provide insights to the puzzle of the dark energy problem [20]. 
Coalescing binary compact objects (CBC) are among the most promising gravitational wave sources. There are two common methods to determine the parameter estimation accuracy for such gravitational wave signals. One is the Fisher information matrix (FIM) method, which provides the Cramer-Rao bound. The other is the Markov chain Monte Carlo (MCMC) method [21] which gives a tighter accuracy estimate than the Fisher information matrix method. The Fisher matrix method is valid for high signalto-noise ratio cases, while the MCMC method is free of this limitation.

In [8], the authors used the Fisher matrix method to do the parameter estimation of the lensed gravitational wave signal for LISA. Their method is guaranteed by the high signal to noise ratio (SNR) of LISA. Also, because the detection event rate for LISA is quite high, it is not interesting to consider the possible detection loss due to using an unlensed gravitational wave template to analyze lensed wave. Differently, for the coming generations of ground based gravitational wave detectors, the detection event rate is not high. So the possible signal loss due to improper usage of gravitational wave form template deserves to be considered. Due to the low SNR, the MCMC method is necessary to ground based detectors for parameter estimation. In this paper we will consider the lensed gravitational wave signals which may be detected by Advanced LIGO and ET detectors. First, we will compare the lensed gravitational wave form to the unlensed one in detail. This comparison gives us some hints on the possible signal loss if we only use templates without lensing corrections for lensed signal. Then we will use MCMC to estimate how much information we can extract from the lensed gravitational wave signal if we use templates with lensing effects.

The arrangement of this paper is as follows. We will describe the wave form model with and without lensing effects in the next section. The instruments' features are also presented there. After that, we compare the lensed wave to an unlensed one in detail in Sec. III. We calculate the corresponding SNR by using the unlensed gravitational wave template to treat the lensed signal. Then possible SNR loss is analyzed and we find that the unlensed wave form is not good enough to treat the lensed gravitational wave. Such SNR loss gives implications to possible signal loss for improper template usage. In Sec. IV we focus on the effects of lensing on the parameter extraction in gravitational wave detection. We begin with the brief introduction of the data analysis methods we adopted in this paper with the nonlensing wave forms. Following that, we investigate how much information we can get if we take the lensed wave form template into consideration to search for the lensed signal. We consider binary compact object parameters and lens parameters separately first. Then we analyze the whole set of parameters. Special attention is paid to the correlation between the binary compact object parameters and lensing parameters. Interestingly, we find that the correlation is negligible. This implies that we can treat these two groups of parameters separately which can largely simplify the data analysis for lensed gravitational wave. Last, we summarize our results and give some discussions in Sec. V.

Throughout this paper we will use units $c=G=1$.

\section{GRAVITATIONAL WAVE FORM MODELS WITH AND WITHOUT LENSING FOR COMPACT BINARIES}

In this paper we use the TaylorF2 wave form [22] (we will refer to it as PN model afterwards) to model the binary black hole, which is also called restricted wave form model [23]. This model can even describe binaries with a neutron star as a binary component [24] for the inspiralling stage when the deformation effect is negligible. More specifically the wave form is written as

$$
h(f)=C \frac{\mathcal{M}^{5 / 6}}{D_{\text {eff }}} f^{-7 / 6} e^{i \Psi(f)},
$$

where $f$ is the frequency, $C$ is some constant relating to observation time, $\mathcal{M} \equiv M \nu^{3 / 5}$ is the chirp mass with $M$ the total mass and $\nu$ the symmetric mass ratio of the binary, $D_{\text {eff }}$ is the effective distance and $\Psi$ is the phase. The explicit expressions for $D_{\text {eff }}$ and $\Psi(f)$ are listed in Appendix A. $C$ is determined by the signal-to-noise ratio (SNR). Following [25] we set $\rho=10$ in this paper for both advanced LIGO detector and ET detector.

There are nine parameters $\left(D, t_{c}, \phi_{c}, M, \nu, \theta, \phi, l, \psi\right)$ in this wave form model. The physical meanings of these parameters are explained in Appendix A. The parameters $(D, \theta, \phi, l, \psi)$ are hidden in our SNR assumption, so we are only concerned with four parameters $\left(t_{c}, \phi_{c}, M, \nu\right)$. Following [25] we use parameters $\left(t_{c}, \phi_{c}, \mathcal{M}, \nu\right)$ instead. We will choose $t_{c}=\phi_{c}=0$ without losing any generalities for this paper. We consider $M=20 M_{\odot}$, and $\nu=0.25$ for a binary black hole with $\mathcal{M} \approx 8.7 M_{\odot}$ and component mass $10 M_{\odot}$ and $10 M_{\odot} ; M=11.4 M_{\odot}$, and $\nu \approx 0.1077$ for a black hole-neutron star binary with $\mathcal{M} \approx 2.99 M_{\odot}$ and component mass $10 M_{\odot}$ for the black hole and $1.4 M_{\odot}$ for the neutron star. This setting is motivated by radio and x-ray observations in our own Galaxy which have strongly indicated that the mass distribution of neutron stars in binaries is sharply peaked around $1.4 M_{\odot}$. We set $M=2.8 M_{\odot}$, and $\nu=0.25$ for neutron star binary with $\mathcal{M} \approx 1.22 M_{\odot}$ and component mass $1.4 M_{\odot}$ for both neutron stars.

Due to the relatively short wavelengths of electromagnetic radiation, the lensing effects of light mainly behave as a light bending effect. For gravitational wave, due to its long wavelengths, it behaves more like sound waves. Compared to the lensing effects on light, the lensing effects on gravitational wave include both the interference effect and the diffraction effect. With the wave optics method, 
one can express the effects of lensing on gravitational wave with a complex amplification factor as [8]

$$
h^{L}(f)=F(f) h(f),
$$

where $h^{L}(f)$ and $h(f)$ denote the lensed wave and unlensed wave in frequency domain respectively. $F(f)$ is the complex amplification factor of lensing effect. For the time domain wave form $h(t)$, we have to transform it to frequency domain through Fourier transformation. Then multiply the resultant wave form with this complex amplification factor. Finally transform the resulted wave form in frequency domain back to time domain. Since we have adopted the frequency domain wave form model in this paper, we do not need to follow this procedure. For lens object, we consider both point mass-type model and singular isothermal sphere (SIS) model in this paper. The explicit amplification factors for these two lensing models are described in Appendix B. If the lensing effect is found important for observation, it is straightforward to generalize our results to more complicated lens models with the method of [9].

There are two parameters describing lens objects, the effective mass of the lens object $M_{l z}$ and the normalized distance $y$ between the lens object and the joining line of the Earth and the gravitational wave source. The normalization is done with respect to the critical radius of the lens object. We refer our readers to [8] for detailed explanations of these two parameters. Parameter $y$ plays the role of impact distance and determines the strength of the lensing effect. Roughly $y<1$ corresponds to strong lensing in which case multiple images will appear.

We consider three types of lensing objects which correspond to stellar mass black hole, intermediate massive black hole and super massive black hole. We use $M_{l z}=$ $10 M_{\odot}$ as a typical example of stellar mass black hole lens object. In practice Cyg X-1 $\left(8.7 M_{\odot}\right)$ may act as this type of lensing case. There are several possible pathways to intermediate massive black hole formation in a star cluster [26-28]. Some observational candidates for an intermediate massive black hole exist [29-31]. Here we use $1000 M_{\odot}$ as a representative example to study intermediate massive black holes as lens objects. For supermassive black hole, we have SgrA* hosted in our Milky Way Galaxy which may play the role of lens object acting on the gravitational wave of binary compact objects for AdvLIGO and ET to detect. So we set $M_{l z}=4.4 \times 10^{6} M_{\odot}$ for this situation.

We simulate the signal as $s(f)=A g(f)+n(f)$, where $A$ is the expectation of the SNR factor, $n(f)$ is the Fourier transform of the noise, and $g(f)$ is the normalized wave form with respect to the inner product,

$$
\left(h_{1}(f), h_{2}(f)\right) \equiv 4 \Re \int_{f_{\min }}^{f_{\max }} \frac{h_{1} \bar{h}_{2}}{S_{n}} d f,
$$

with $f_{\min }$ and $f_{\max }$ the lower and upper frequency bound of the detector in question, and $S_{n}$ is the one-sided noise power spectrum density (PSD). The overbar denotes the complex conjugate. We use the data "ZERO_DET_high_P.txt" [32] as the $S_{n}$ for Advanced LIGO. For Einstein telescope we adopt the following PSD [33]:

$$
\begin{gathered}
\sqrt{S}=10^{-25}\left[2.39 \times 10^{-27} x^{-15.64}+0.349 x^{-2.145}\right. \\
\left.+1.76 x^{-0.12}+0.409 x^{1.1}\right] \\
x=f / 100
\end{gathered}
$$

\section{SNR LOSS FOR LENSED GRAVITATIONAL WAVE SIGNAL WITH UNLENSED TEMPLATE}

Until now, the lensing effect on compact-binarycoalescence (CBC) signals has not been thoroughly studied for ground-based GW detectors. Ignoring such effects in the searching wave form templates may cause SNR loss and lead to systematic bias in the parameter estimation. In this section, we will investigate these effects in detail.

The amplitude comparison between the unlensed wave form and the lensed wave form corresponding to stellar mass lens object, intermediate massive lens object and supermassive lens object for AdvLIGO are shown in Fig. 1. For ET the results are similar to Fig. 1 up to 1 order smaller amplitude. The main features shown in Fig. 1 depend on the wave form only and not on the properties of the detector. The SIS lensing model results in quite similar plots as that of the point lensing model shown here. For both the point lensing model and the SIS lensing model, the lensing effect can be neglected if $w=2 \pi M_{l z} f<1$, because the complex amplifier of the lensing effect is roughly 1 in this situation [8]. So we expect the lensing effect for $M_{l z}=10 M_{\odot}$ appears around $f \sim 10^{4} \mathrm{~Hz}$. This explains why the two lines for $M_{l z}=10 M_{\odot}$ in Fig. 1 show little difference. So we do not need to consider the lensing effect from the stellar mass black hole for AdvLIGO and ET detection. For $M_{l z}=1000 M_{\odot}$ we expect the lensing effect will start to show up around $100 \mathrm{~Hz}$. From Fig. 1 we can see that this is exactly the case. The amplitude modulation shows up at about $100 \mathrm{~Hz}$ for all the BH-BH, BH-NS and NS-NS cases. Interestingly, the signal in the sensitive frequency range of LIGO (around $100 \mathrm{~Hz}$ ) is increased a little bit by lensing. Regarding $M_{l z}=4.4 \times 10^{6} M_{\odot}$, we expect the lensing effect will start to be important at $0.1 \mathrm{~Hz}$. Because we have $w=2 \pi M_{l z} f \geq 100$ for the whole AdvLIGO and ET frequency range, the optical approximation for the lensing model can work well in this case. In Fig. 1 we do see that for the whole frequency band of AdvLIGO there is a significant amplitude modulation which comes from lensing. This kind of modulation can be used to extract the information of the supermassive black hole, which has already been pointed out in [34]. We will analyze this extraction quantitatively in the next section.

In order to estimate the possible detection loss when we use the unlensed wave form template to treat the lensed signal, we calculate the fitting factor between the unlensed 

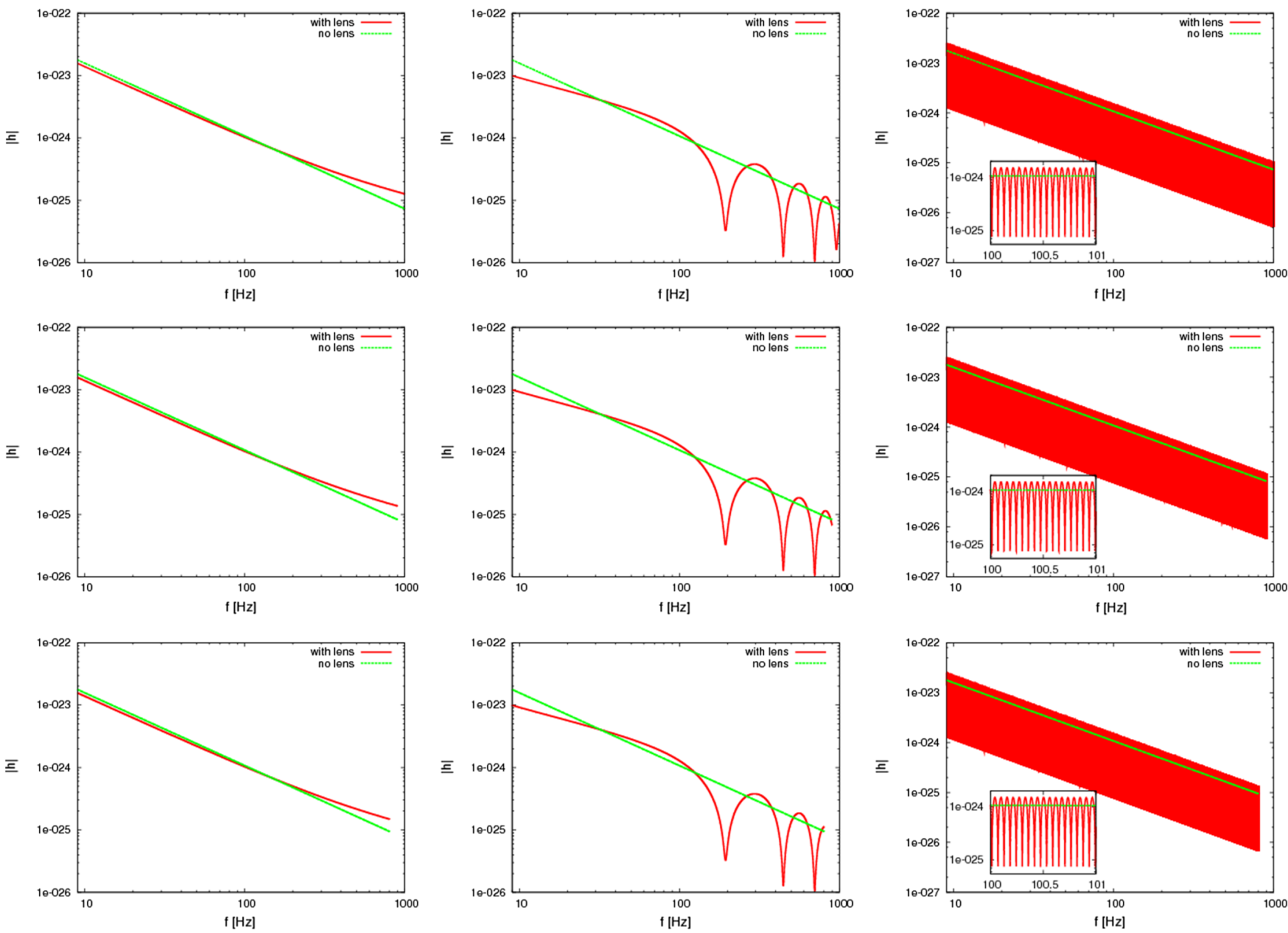

FIG. 1 (color online). Amplitude comparison of the lensed wave form to the unlensed wave form. We have set SNR $=10$ for AdvLIGO and $y=0.1$ for lensing strength. From the top row to the bottom row: The simulated signals for BH-BH, BH-NS and NS-NS binaries respectively. From the left column to the right column: The lens mass $M_{l z}$ equals to $10 M_{\odot}, 1000 M_{\odot}$ and $4.4 \times 10^{6} M_{\odot}$ respectively. The subplots in the right column are the corresponding zoom-in for the frequency range [100,101]. For the $x$ axis, we have used log scale in the main plots while have used linear scale in the zoom-in plots of right column. For all of the $y$ axis log scale is used.

wave form and the lensed one. Based on the inner product (3), we define the fitting factor as [35]

$$
F F \equiv \max _{t_{c}, \phi_{c}, \mathcal{M}, \nu} \frac{\left(h_{u}\left(t_{c}, \phi_{c}, \mathcal{M}, \nu\right) \mid h_{l}\right)}{\sqrt{\left(h_{u} \mid h_{u}\right)\left(h_{l} \mid h_{l}\right)}},
$$

where $h_{l}$ represents the lensed gravitational wave corresponding to some specific parameters $\left(t_{c 0}, \phi_{c 0}, \mathcal{M}_{0}, \nu_{0}\right.$; $\left.M_{l z 0}, y_{0}\right)$. The maxima is obtained by varying unlensed wave form $h_{u}$ template parameters $\left(t_{c}, \phi_{c}, \mathcal{M}, \nu\right)$. There is no simulated noise in either $h_{u}$ or $h_{l}$. Let us denote the parameters which correspond to the maxima as $\left(t_{c u}, \phi_{c u}\right.$, $\left.\mathcal{M}_{u}, \nu_{u}\right)$. Then we define the parameters detection bias as

$$
\begin{gathered}
E_{M} \equiv \frac{\left|\mathcal{M}_{0}-\mathcal{M}_{u}\right|}{\mathcal{M}_{0}}, \\
E_{\nu} \equiv \frac{\left|\nu_{0}-\nu_{u}\right|}{\nu_{0}} .
\end{gathered}
$$

First, we check the fitting factor and parameters bias for the CBC source with lensing mass $M_{l z}=10 M_{\odot}$ in Table I.
In this table, we used the Advanced LIGO detector. The results for the ET detector are similar. As we expect, the lensing effect is negligible for $M_{l z}=10 M_{\odot}$ in strong lensing cases where $y<1$. All of the fitting factors are bigger than 0.97, and the parameters bias are less than $0.04 \%$. This result quantitatively confirms the conclusion we obtained above, which says that the lensing effect introduced by solar massive lensing objects for ground based detectors is negligible.

Then we check the lensing effect with $M_{l z}=1000 M_{\odot}$ and $M_{l z}=4.4 \times 10^{6} M_{\odot}$ in Tables II and III. We find that the fitting factor may decrease to about 0.8 for $M_{l z}=$ $1000 M_{\odot}$ and may decrease more to about 0.7 for $M_{l z}=$ $4.4 \times 10^{6} M_{\odot}$. At the same time we also note that the parameters bias is still negligible. Among all of the cases, the largest bias is less than $0.5 \%$. This is because the mass and mass ratio parameters are derived by matching the frequency evolution for thousands of cycles, and that will not change much because of the lensing magnification. 
TABLE I. The fitting factor and the parameters bias for lensing mass $M_{l z}=10 M_{\odot}$. The Advanced LIGO detector is used here.

\begin{tabular}{llccc}
\hline \hline Model & CBC & FF & $E_{M}$ & $E_{\nu}$ \\
\hline Point lens & BH-BH & 0.986 & $0.026 \%$ & $0.001 \%$ \\
& BH-NS & 0.986 & $0.009 \%$ & $0.027 \%$ \\
$y=0.01$ & NS-NS & 0.994 & $0.001 \%$ & $0.001 \%$ \\
Point lens & BH-BH & 0.988 & $0.026 \%$ & $0.001 \%$ \\
& BH-NS & 0.988 & $0.009 \%$ & $0.026 \%$ \\
$y=0.03$ & NS-NS & 0.994 & $0.001 \%$ & $0.001 \%$ \\
Point lens & BH-BH & 0.989 & $0.022 \%$ & $0.001 \%$ \\
& BH-NS & 0.989 & $0.009 \%$ & $0.023 \%$ \\
$y=0.1$ & NS-NS & 0.995 & $0.001 \%$ & $0.001 \%$ \\
Point lens & BH-BH & 0.987 & $0.017 \%$ & $0.001 \%$ \\
& BH-NS & 0.987 & $0.011 \%$ & $0.024 \%$ \\
$y=0.3$ & NS-NS & 0.996 & $0.001 \%$ & $0.001 \%$ \\
SIS lens & BH-BH & 0.992 & $0.038 \%$ & $0.001 \%$ \\
& BH-NS & 0.992 & $0.009 \%$ & $0.007 \%$ \\
$y=0.01$ & NS-NS & 0.992 & $0.001 \%$ & $0.001 \%$ \\
SIS lens & BH-BH & 0.992 & $0.038 \%$ & $0.001 \%$ \\
& BH-NS & 0.992 & $0.007 \%$ & $0.023 \%$ \\
$y=0.03$ & NS-NS & 0.992 & $0.001 \%$ & $0.001 \%$ \\
SIS lens & BH-BH & 0.992 & $0.035 \%$ & $0.001 \%$ \\
$y=0.1$ & BH-NS & 0.993 & $0.008 \%$ & $0.024 \%$ \\
SIS lens & NS-NS & 0.993 & $0.001 \%$ & $0.001 \%$ \\
$y=0.3$ & BH-BH & 0.972 & $0.017 \%$ & $0.001 \%$ \\
\hline \hline & BH-NS & 0.972 & $0.011 \%$ & $0.023 \%$ \\
& NS-NS & 0.994 & $0.001 \%$ & $0.001 \%$ \\
\hline & & & &
\end{tabular}

TABLE II. The fitting factor and the parameters bias for lensing mass $M_{l z}=1000 M_{\odot}$. The Advanced LIGO detector is used here.

\begin{tabular}{llccc}
\hline \hline Model & CBC & FF & $E_{M}$ & $E_{\nu}$ \\
\hline Point lens & BH-BH & 0.954 & $0.048 \%$ & $0.004 \%$ \\
& BH-NS & 0.955 & $0.023 \%$ & $0.023 \%$ \\
$y=0.01$ & NS-NS & 0.955 & $0.002 \%$ & $0.001 \%$ \\
Point lens & BH-BH & 0.973 & $0.048 \%$ & $0.001 \%$ \\
& BH-NS & 0.954 & $0.023 \%$ & $0.024 \%$ \\
$y=0.03$ & NS-NS & 0.950 & $0.009 \%$ & $0.001 \%$ \\
Point lens & BH-BH & 0.832 & $0.157 \%$ & $0.001 \%$ \\
& BH-NS & 0.833 & $0.040 \%$ & $0.024 \%$ \\
$y=0.1$ & NS-NS & 0.833 & $0.006 \%$ & $0.001 \%$ \\
Point lens & BH-BH & 0.792 & $0.024 \%$ & $0.002 \%$ \\
& BH-NS & 0.792 & $0.011 \%$ & $0.023 \%$ \\
$y=0.3$ & NS-NS & 0.792 & $0.001 \%$ & $0.001 \%$ \\
SIS lens & BH-BH & 0.954 & $0.069 \%$ & $0.004 \%$ \\
$y=0.01$ & BH-NS & 0.954 & $0.027 \%$ & $0.023 \%$ \\
SIS lens & NS-NS & 0.955 & $0.003 \%$ & $0.001 \%$ \\
& BH-BH & 0.974 & $0.038 \%$ & $0.001 \%$ \\
$y=0.03$ & BH-NS & 0.954 & $0.022 \%$ & $0.023 \%$ \\
SIS lens & NS-NS & 0.950 & $0.010 \%$ & $0.001 \%$ \\
$y=0.1$ & BH-BH & 0.832 & $0.523 \%$ & $0.001 \%$ \\
SIS lens & BH-NS & 0.829 & $0.042 \%$ & $0.024 \%$ \\
$y=0.3$ & NS-NS & 0.829 & $0.006 \%$ & $0.001 \%$ \\
\hline \hline & BH-BH & 0.800 & $0.028 \%$ & $0.001 \%$ \\
& BH-NS & 0.800 & $0.008 \%$ & $0.024 \%$ \\
& NS-NS & 0.800 & $0.001 \%$ & $0.001 \%$ \\
\hline & & & & \\
& & & & \\
& & & &
\end{tabular}

TABLE III. The fitting factor and the parameters bias for lensing mass $M_{l z}=4.4 \times 10^{6} M_{\odot}$. The Advanced LIGO detector is used here.

\begin{tabular}{|c|c|c|c|c|}
\hline Model & $\mathrm{CBC}$ & $\mathrm{FF}$ & $E_{M}$ & $E_{\nu}$ \\
\hline \multirow[t]{2}{*}{ Point lens } & BH-BH & 0.711 & $0.001 \%$ & $0.001 \%$ \\
\hline & BH-NS & 0.711 & $0.013 \%$ & $0.023 \%$ \\
\hline$y=0.01$ & NS-NS & 0.711 & $0.001 \%$ & $0.001 \%$ \\
\hline \multirow[t]{2}{*}{ Point lens } & BH-BH & 0.718 & $0.001 \%$ & $0.001 \%$ \\
\hline & BH-NS & 0.718 & $0.013 \%$ & $0.023 \%$ \\
\hline$y=0.03$ & NS-NS & 0.718 & $0.001 \%$ & $0.001 \%$ \\
\hline \multirow[t]{2}{*}{ Point lens } & BH-BH & 0.741 & $0.001 \%$ & $0.001 \%$ \\
\hline & BH-NS & 0.741 & $0.013 \%$ & $0.023 \%$ \\
\hline$y=0.1$ & NS-NS & 0.741 & $0.001 \%$ & $0.001 \%$ \\
\hline \multirow[t]{2}{*}{ Point lens } & BH-BH & 0.803 & $0.001 \%$ & $0.001 \%$ \\
\hline & BH-NS & 0.803 & $0.013 \%$ & $0.023 \%$ \\
\hline$y=0.3$ & NS-NS & 0.803 & $0.001 \%$ & $0.001 \%$ \\
\hline \multirow{2}{*}{ SIS lens } & BH-BH & 0.711 & $0.001 \%$ & $0.001 \%$ \\
\hline & BH-NS & 0.711 & $0.013 \%$ & $0.023 \%$ \\
\hline$y=0.01$ & NS-NS & 0.711 & $0.001 \%$ & $0.001 \%$ \\
\hline \multirow[t]{2}{*}{ SIS lens } & BH-BH & 0.718 & $0.001 \%$ & $0.001 \%$ \\
\hline & BH-NS & 0.718 & $0.013 \%$ & $0.023 \%$ \\
\hline$y=0.03$ & NS-NS & 0.718 & $0.001 \%$ & $0.001 \%$ \\
\hline \multirow[t]{2}{*}{ SIS lens } & BH-BH & 0.741 & $0.001 \%$ & $0.001 \%$ \\
\hline & BH-NS & 0.741 & $0.013 \%$ & $0.023 \%$ \\
\hline$y=0.1$ & NS-NS & 0.741 & $0.001 \%$ & $0.001 \%$ \\
\hline \multirow[t]{2}{*}{ SIS lens } & BH-BH & 0.803 & $0.001 \%$ & $0.001 \%$ \\
\hline & BH-NS & 0.803 & $0.013 \%$ & $0.023 \%$ \\
\hline$y=0.3$ & NS-NS & 0.803 & $0.001 \%$ & $0.001 \%$ \\
\hline
\end{tabular}

The corresponding SNR by using the PN wave form model (1) as a template while using the lensed gravitational wave together with instrumental noise as the signal roughly equals the fitting factor times the real SNR of the lensed signal. We have also confirmed this relation through numerical simulation. Based on this relation, the above fitting factor results imply that the lensing effect affects significantly on the gravitational waves emitted from binary compact objects. The resulted SNR may decrease $20 \%$ for lensing mass $M_{l z}=1000 M_{\odot}$. For lensing mass $M_{l z}=$ $4.4 \times 10^{6} M_{\odot}$ the SNR may decrease even $30 \%$. Therefore using the unlensed template to treat the lensed signal may result in significant signal loss. For example, to ET the event rates may decrease from tens per year [19] to less than ten for lensing mass $M_{l z}=1000 M_{\odot}$ and to roughly one per year for lensing mass $M_{l z}=4.4 \times 10^{6} M_{\odot}$. Regarding Advanced LIGO, the unlensed template may turn the lensing detection from possible to impossible.

\section{PARAMETER ESTIMATION OF LENSED GRAVITATIONAL WAVE FOR COMPACT BINARIES}

\section{A. Parameter estimation methods}

In this paper, we only concentrate on the parameters estimate accuracy. So we use the true value for the 
parameters as the starting point for MCMC chains. This setting saves us the burn-in stage of the simulation.

Both the unlensed gravitational wave form model (1) and the lensed one (2) can be written as

$$
h\left(f ; \Theta, t_{c}, \phi_{c}\right)=H(f ; \Theta) e^{i 2 \pi f t_{c}-i \phi_{c}},
$$

where $\Theta$ denotes the rest of the wave form parameters excluding $t_{c}$ and $\phi_{c}$. The log likelihood (up to a constant) is

$$
\begin{aligned}
\Lambda\left(\Theta, t_{c}, \phi_{c}\right) & =\left(s, h\left(\Theta, t_{c}, \phi_{c}\right)\right), \\
& =4 \Re \int_{f_{\min }}^{f_{\max }} \frac{\bar{s} H}{S_{n}} e^{i 2 \pi f t_{c}-i \phi_{c}} d f .
\end{aligned}
$$

The constant phase $\phi_{c}$ and the coalescence time $t_{c}$ can be maximized over as follows:

$$
\begin{aligned}
\Lambda(\Theta) & \equiv \max _{t_{c}, \phi_{c}} \Lambda\left(\Theta, t_{c}, \phi_{c}\right), \\
& =4 \max _{t_{c}}\left|\int_{f_{\min }}^{f_{\max }} \overline{\bar{s} H} e_{n}^{i 2 \pi f t_{c}} d f\right|, \\
& \approx 2 \max _{t_{c}}\left|\int_{-\infty}^{\infty} \frac{\bar{s} H}{S_{n}} e^{i 2 \pi f t_{c}} d f\right|,
\end{aligned}
$$

which can be simply approximated by twice the largest absolute value of the Fourier transform of $\frac{\bar{s} H}{S_{n}}$. This technique is called F-statistics [36]. This scheme has been widely adopted in gravitational wave data analysis, and we will use this method to eliminate the parameters $t_{c}$ and $\phi_{c}$ from our parameters list. For MCMC algorithms, we calculate the transition probability through

$$
p\left(\Theta_{2} \mid \Theta_{1}\right)=e^{\Lambda\left(\Theta_{2}\right)-\Lambda\left(\Theta_{1}\right)} .
$$

Based on the above transition probability, the MetropolisHastings scheme is adopted. In our implementation of MCMC, we employ $5 \times 10^{5}$ or $2 \times 10^{6}$ iterations for different cases to make sure the chains converge. During the simulation we use the proposal distribution

$$
\prod_{i} \frac{1}{\sqrt{2 \pi \epsilon_{i}}} e^{-\frac{\left(\theta_{i}-\theta_{i}^{(n)}\right)^{2}}{2 \epsilon_{i}^{2}}},
$$

where $\theta_{i}^{(n)}$ is the parameter at the $n$th step and $\theta_{i}$ is the proposed parameter. We set $\epsilon_{i}$ equal to the tenth of the estimated measurement errors through the Fisher matrix method.

Regarding the Fisher matrix, since we mainly concern the parameters $M$ and $\nu$, we can use the projector method to reduce $t_{c}$ and $\phi_{c}$,

$$
\bar{F}_{i j} \equiv F_{i j}-F_{i a} \hat{F}^{a b} F_{b j},
$$

where $i$ and $j$ run over $M$ and $\nu$, and $a$ and $b$ run over $t_{c}$ and $\phi_{c} . F$ is the full Fisher matrix for four parameters, $\hat{F}$ is the block matrix of $F$ for $t_{c}$ and $\phi_{c}$ parts, and $\bar{F}$ is the reduced
TABLE IV. With the Fisher matrix and projector method (14) we estimate the parameter extraction errors. Here we have assumed SNR $=10$.

\begin{tabular}{llll}
\hline \hline IFO & CBC & $\Delta \mathcal{M} / \mathcal{M}$ & \multicolumn{1}{c}{$\Delta \nu$} \\
\hline \multirow{4}{*}{ AdvLIGO } & BH-BH & $0.084 \%$ & 0.0025 \\
& BH-NS & $0.014 \%$ & 0.00043 \\
& NS-NS & $0.0033 \%$ & 0.0013 \\
ET & BH-BH & $0.040 \%$ & 0.0017 \\
\hline \hline & BH-NS & $0.0080 \%$ & 0.00028 \\
& NS-NS & $0.0029 \%$ & 0.0016 \\
\hline
\end{tabular}

Fisher matrix for the intrinsic parameters $M$ and $\nu$. Here we have used $\hat{F}^{a b}$ to denote the inverse matrix of $\hat{F}_{a b}$ and the Einstein summation rule is also adopted. For later comparison we list the results in Table IV. Our result is consistent to the parameter estimation in [25].

We use the parameters $\mathcal{M}$ and $\nu$ in the MCMC simulations. We have checked the chain traces. As we expected, the trace of SNR oscillates below but around the setting value 10 , and the parameters $\mathcal{M}$ and $\nu$ oscillate around the true values. In Fig. 2 we plot out the probability density function (PDF). In this plot we also mark out the $1-\sigma$ parameter region for both Fisher matrix method and MCMC method. The $1-\sigma$ parameter region is defined by $\theta^{T}\left(F^{-1}\right) \theta=1$, where $F$ is the Fisher information matrix and $\theta$ represents the vector composed with the parameters in question. This corresponds to the probability $68.3 \%$ in the one-dimensional case and $39.3 \%$ in the twodimensional case respectively. For ET, the $1-\sigma$ parameter regions for these two methods are roughly the same. But for AdvLIGO, we find that the Fisher matrix method underestimates such region a little bit. This result is consistent to [37]. The most possible parameters $(\mathcal{M}, \nu)$ got by MCMC are $\left(8.704 M_{\odot}, 0.2496\right),\left(2.994 M_{\odot}, 0.1076\right),\left(1.219 M_{\odot}\right.$, 0.2497) for BH-BH, BH-NS, NS-NS with AdvLIGO, and $\left(8.706 M_{\odot}, \quad 0.2497\right), \quad\left(2.994 M_{\odot}, \quad 0.1077\right), \quad\left(1.219 M_{\odot}\right.$, 0.2498) for BH-BH, BH-NS, NS-NS with ET.

Through reducing the full PDF to marginal PDF, we can estimate the parameters measurement errors which are listed in Table V. Compared to Table IV we can see that for advLIGO the MCMC errors in the chirp mass determination are roughly a factor 3-4 higher than FIM estimates, whereas they are in general comparable for ET. The errors in mass ratio for MCMC and FIM are roughly the same for both AdvLIGO and ET. In this kind of situation, MCMC results are more trustable [37,38]. In the following analysis of this paper we will use MCMC to do a detailed estimate while applying the Fisher matrix method to give a guideline for the analysis.

We have seen the importance of including lensing corrections to the templates to analyze the lensed signal in the above section. In the following we will investigate what kind of information we can get from the lensed signal if we use lensed templates. As described in the above section, we have six parameters $\left(M_{l z}, y, t_{c}, \phi_{c}, M, \nu\right)$ to be 

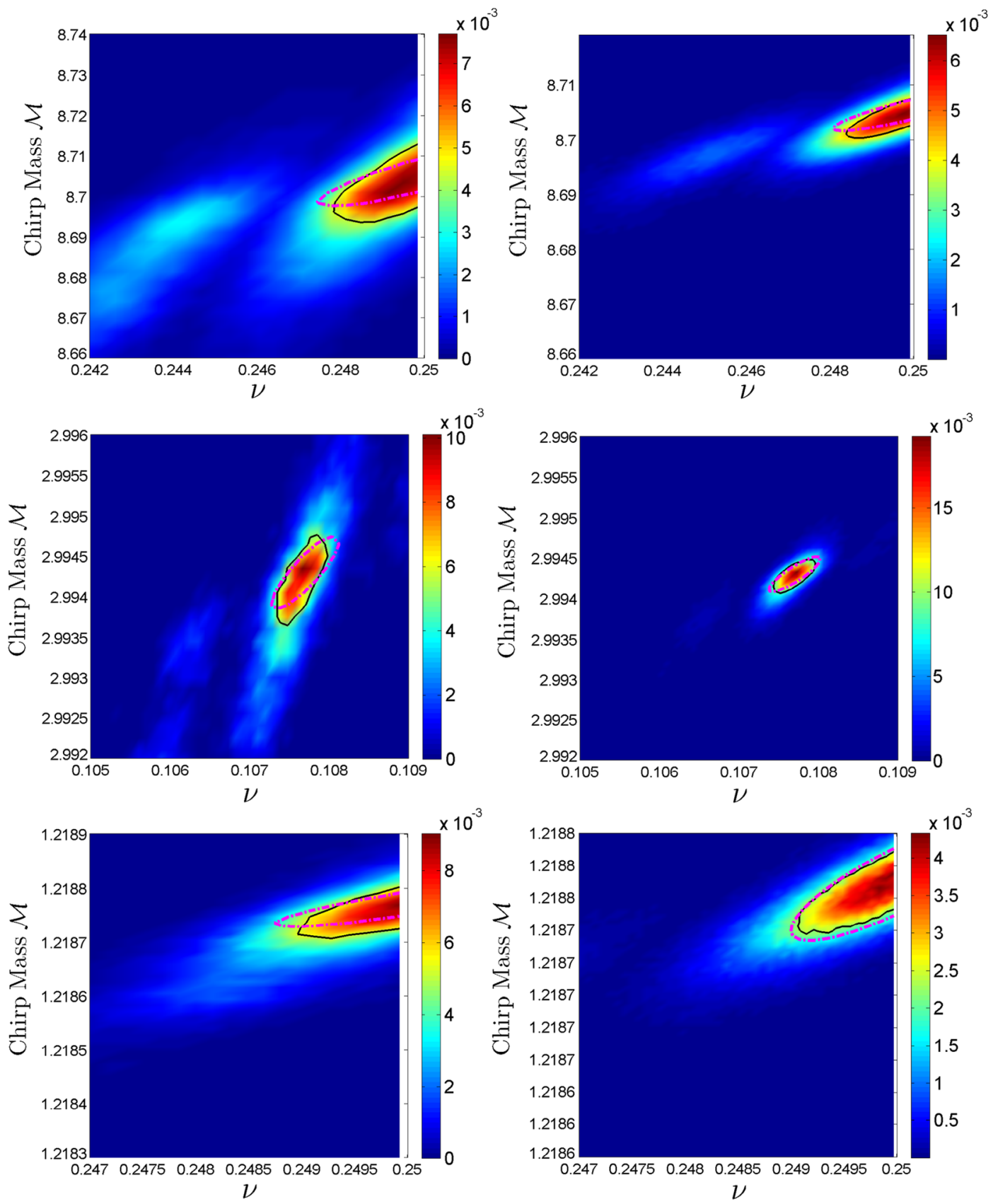

FIG. 2 (color online). Probability density functions (PDF) by MCMC for gravitational wave signal without lensing. The magenta dotdashed line in these plots marks out the $1-\sigma$ parameter region through the Fisher matrix method. The black solid line marks out such a region for the MCMC method. The left plots are for Advanced LIGO, and the right ones are for ET. From the top row to the bottom row the plots correspond to $\mathrm{BH}-\mathrm{BH}, \mathrm{BH}-\mathrm{NS}$ and NS-NS respectively.

estimated for the lensed gravitational wave form model. Because we are treating the strong lensing case, we chose $y=0.1$ as an example for all of the rest of the investigations. First, we will consider how $M_{l z}$ and $y$ affect the parameter estimation for binary compact sources.

\section{B. Fixing lens parameters case}

In this subsection we assume the lens parameters $M_{l z}$ and $y$ are known a priori. First, we use the Fisher matrix method to estimate the extraction error for the parameters $\mathcal{M}$ and $\nu$. We list the results for AdvLIGO and ET in Table VI. Compared to the unlensed case, we find that the errors determination now are in general a factor 2-3 larger than that in the unlensed cases. Especially, the lensing effect makes $\Delta \mathcal{M}$ larger in all situations. This result is consistent to the finding of [8].

In Fig. 3 we plot out the MCMC resulted joint PDFs for $M_{l z}=1000 M_{\odot}$ and point lensing model with AdvLIGO 
TABLE V. With MCMC we estimate the parameter extraction error for gravitational wave model (1). Here we have assumed $\mathrm{SNR}=10$. The errors correspond to the 15.9 and 84.2 percentile of the posterior distribution which gives a $68.3 \%(1 \sigma)$ posterior credibility.

\begin{tabular}{llll}
\hline \hline IFO & CBC & $\Delta \mathcal{M} / \mathcal{M}$ & \multicolumn{1}{c}{$\Delta \nu$} \\
\hline & BH-BH & $0.39 \%$ & 0.0038 \\
\multirow{3}{*}{ AdvLIGO } & BH-NS & $0.064 \%$ & 0.00097 \\
& NS-NS & $0.0094 \%$ & 0.0014 \\
& BH-BH & $0.059 \%$ & 0.0015 \\
ET & BH-NS & $0.013 \%$ & 0.00040 \\
\hline \hline
\end{tabular}

TABLE VI. With the Fisher matrix method we estimate the parameter extraction error for the PN gravitational wave model (1). As in Table IV, we have assumed SNR $=10$. Here we used the point lensing model (B1) and $M_{l z}=1000 M_{\odot}$.

\begin{tabular}{llll}
\hline \hline IFO & CBC & $\Delta \mathcal{M} / \mathcal{M}$ & \multicolumn{1}{c}{$\Delta \nu$} \\
\hline \multirow{3}{*}{ AdvLIGO } & BH-BH & $0.11 \%$ & 0.0031 \\
& BH-NS & $0.046 \%$ & 0.0020 \\
& NS-NS & $0.0045 \%$ & 0.0016 \\
ET & BH-BH & $0.14 \%$ & 0.0039 \\
& BH-NS & $0.025 \%$ & 0.00066 \\
\hline \hline
\end{tabular}

detector. The results for the ET, SIS lensing model and $M_{l z}=4.4 \times 10^{6} M_{\odot}$ are similar. Similar to the results in Fig. 2, the $1-\sigma$ region of parameters for ET is smaller than that for AdvLIGO. Corresponding to Fig. 3, the most possible parameters $(\mathcal{M}, \nu)$ are $\left(8.705 M_{\odot}, 0.2495\right)$, $\left(2.994 M_{\odot}, 0.1075\right),\left(1.219 M_{\odot}, 0.2497\right)$ respectively. The corresponding parameter estimation error is listed in Table VII. Compared to Table V, we find that the measurement error of binary parameters is only mildly affected by lensing. This result confirms the one obtained above via the Fisher matrix method.

\section{Fixing compact binary parameters case}

We have seen in the above subsection that the lensing effect of a known lensing object makes the parameter extraction a little harder for $\mathrm{CBC}$ gravitational wave sources. In this subsection we will check if we can benefit from the lensed signal and get some information of lens object with a known CBC source. To investigate this problem we assume the parameters $\left(t_{c}, \phi_{c}, M, \nu\right)$ are known a priori. We use the Fisher matrix method as a guideline for MCMC simulation to estimate the lens parameters $M_{l z}$ and $y$. We list the result in Table VIII. The relative accuracy of both $M_{l z}$ and $y$ measurement is roughly $20 \%$. Note that $y$ is related to $D_{L}$, the distance to the lensing object, and $D_{S}$, the distance to the binary objects; we expect that one can use the information of $y$ to determine $D_{L}$. This determination will roughly result in $20 \%$ confidence. This kind of error level is comparable to the accuracy level obtained with other methods [39]. This result is consistent to the one in [8].

Since $20 \%$ is dangerously close to $100 \%$, the above result means we can get little information of lens object from the lensed gravitational wave. One may hope that the Fisher matrix overestimates estimation errors a bit, and MCMC might result in smaller estimation errors, but this turns out to be not the case. Through MCMC, we find that the measurement error is even worse. When the lensing parameters are turned on, the MCMC chain converges much slower. Through tests, we find that $2 \times 10^{6}$ iterations are enough for convergence. The uncertainty becomes near $100 \%$. This means we cannot determine $M_{l z}$ and $y$ through lensed gravitational wave detection. But this does not mean we cannot get any information of lens object from the lensed gravitational wave detection. The result for MCMC is more complicated than simple numbers to indicate the measurement error. We plot out the joint PDF gotten by MCMC simulation in Fig. 4. This plot corresponds to $M_{l z}=1000 M_{\odot}$ and the point lensing model. It is for $\mathrm{BH}-\mathrm{BH}$ with the Advanced LIGO detector. The results for ET, $M_{l z}=4.4 \times 10^{6} M_{\odot}$ and the SIS lensing model are
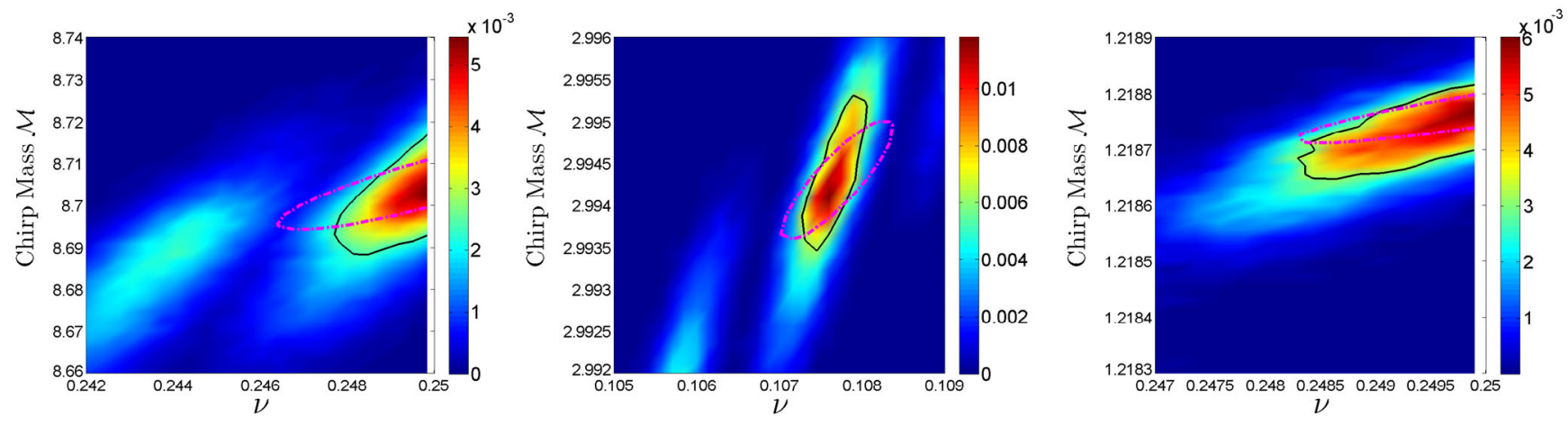

FIG. 3 (color online). Joint PDFs for parameters $\mathcal{M}$ and $\nu$. From left to right the plots correspond to BH-BH, BH-NS and NS-NS respectively. Here we used $M_{l z}=1000 M_{\odot}$ and the point lensing model. These plots are for Advanced LIGO. The plot convention is the same as Fig. 2. 
TABLE VII. With MCMC we estimate the parameter extraction error for the PN gravitational wave model (1) with two different lensing effects. Here we again have assumed $\mathrm{SNR}=10$. The errors correspond to the 15.9 and 84.2 percentile of the posterior distribution which gives a $68.3 \%(1 \sigma)$ posterior credibility.

\begin{tabular}{|c|c|c|c|}
\hline IFO & $\mathrm{CBC}$ & $\Delta \mathcal{M} / \mathcal{M}$ & $\Delta \nu$ \\
\hline \multicolumn{4}{|c|}{ Point lensing source $(\mathrm{B} 1) M_{l z}=1000 M_{\odot}$} \\
\hline & $\mathrm{BH}-\mathrm{BH}$ & $0.38 \%$ & 0.0031 \\
\hline \multirow[t]{3}{*}{ AdvLIGO } & BH-NS & $0.089 \%$ & 0.0014 \\
\hline & NS-NS & $0.012 \%$ & 0.0010 \\
\hline & $\mathrm{BH}-\mathrm{BH}$ & $0.061 \%$ & 0.0017 \\
\hline \multirow[t]{2}{*}{ ET } & BH-NS & $0.016 \%$ & 0.00041 \\
\hline & NS-NS & $0.0021 \%$ & 0.0051 \\
\hline \multicolumn{4}{|c|}{ SIS lensing source $(\mathrm{B} 2) M_{l z}=1000 M_{\odot}$} \\
\hline & $\mathrm{BH}-\mathrm{BH}$ & $0.40 \%$ & 0.0032 \\
\hline \multirow[t]{3}{*}{ AdvLIGO } & BH-NS & $0.094 \%$ & 0.0011 \\
\hline & NS-NS & $0.0094 \%$ & 0.0036 \\
\hline & $\mathrm{BH}-\mathrm{BH}$ & $0.0064 \%$ & 0.0012 \\
\hline \multirow[t]{2}{*}{ ET } & BH-NS & $0.016 \%$ & 0.0038 \\
\hline & NS-NS & $0.0026 \%$ & 0.00051 \\
\hline \multicolumn{4}{|c|}{ Point lensing source (B1) $M_{l z}=4.4 \times 10^{6} M_{\odot}$} \\
\hline & $\mathrm{BH}-\mathrm{BH}$ & $0.32 \%$ & 0.0031 \\
\hline \multirow[t]{3}{*}{ AdvLIGO } & $\mathrm{BH}-\mathrm{NS}$ & $0.084 \%$ & 0.0011 \\
\hline & NS-NS & $0.0096 \%$ & 0.0012 \\
\hline & $\mathrm{BH}-\mathrm{BH}$ & $0.063 \%$ & 0.0016 \\
\hline \multirow[t]{2}{*}{ ET } & BH-NS & $0.012 \%$ & 0.00042 \\
\hline & NS-NS & $0.0023 \%$ & 0.00061 \\
\hline \multicolumn{4}{|c|}{ SIS lensing source (B2) $M_{l z}=4.4 \times 10^{6} M_{\odot}$} \\
\hline & $\mathrm{BH}-\mathrm{BH}$ & $0.33 \%$ & 0.0039 \\
\hline \multirow[t]{3}{*}{ AdvLIGO } & BH-NS & $0.069 \%$ & 0.0011 \\
\hline & NS-NS & $0.0096 \%$ & 0.0015 \\
\hline & $\mathrm{BH}-\mathrm{BH}$ & $0.062 \%$ & 0.0015 \\
\hline \multirow[t]{2}{*}{ ET } & BH-NS & $0.015 \%$ & 0.0043 \\
\hline & NS-NS & $0.0024 \%$ & 0.0060 \\
\hline
\end{tabular}

TABLE VIII. With the Fisher matrix method we estimate the parameter measurement error for the PN gravitational wave model (1). We have again assumed $\mathrm{SNR}=10$. Different to Table VI, we fix $t_{c}, \phi_{c}, \mathcal{M}$ and $\nu$, and estimate lensing parameters $\Delta M_{l z} / M_{l z}$ and $\Delta y$. Here we used point lensing model (B1) and $M_{l z}=1000 M_{\odot}$.

\begin{tabular}{lccc}
\hline \hline IFO & CBC & $\Delta M_{l z} / M_{l z}$ & $\Delta y$ \\
\hline \multirow{3}{*}{ AdvLIGO } & BH-BH & $20 \%$ & 0.020 \\
& BH-NS & $21 \%$ & 0.020 \\
& NS-NS & $20 \%$ & 0.020 \\
ET & BH-BH & $20 \%$ & 0.020 \\
& BH-NS & $20 \%$ & 0.020 \\
& NS-NS & $20 \%$ & 0.020 \\
\hline \hline
\end{tabular}

similar. From this plot we can see that the PDF ranges along all of the parameters. That explains why the parameter extraction error mentioned above is large. Also we notice that the PDF is limited in a narrow band, which means the lensed signal gives us very stringent correlation relation

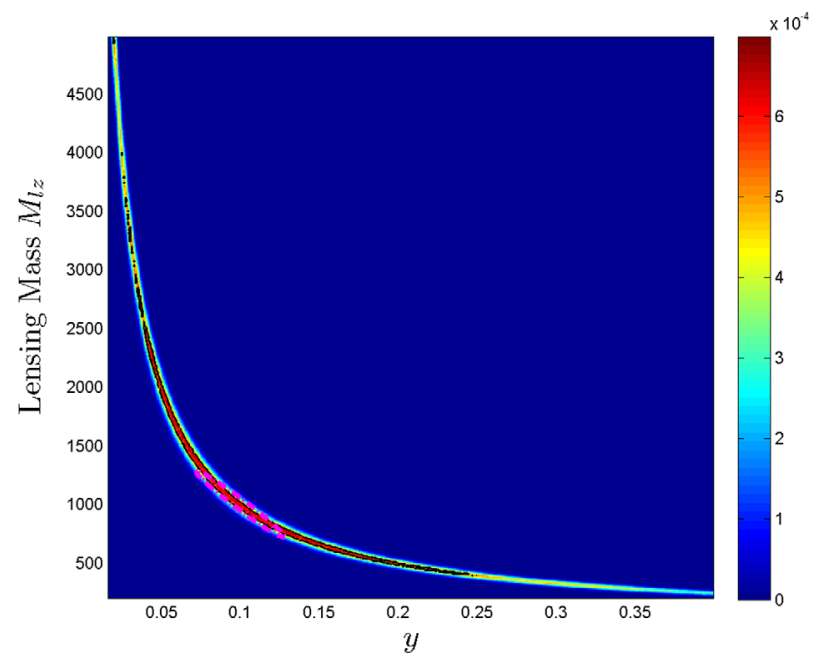

FIG. 4 (color online). Joint PDFs for the parameters $M_{l z}$ and $y$. Here we used $M_{l z}=1000 M_{\odot}$ and the point lensing model. This plot is for BH-BH with the Advanced LIGO detector. The plot convention is the same as Fig. 2.

between lens mass $M_{l z}$ and lensing strength parameter $y$. The combination of this correlation information and other observation result may do better science. For example, the optical observation may give us the mass of the lens object. With the mass $M_{l z}$, looking through the correlation curve, we may extract some parameters accurately, say the distance parameter $D_{L}$. This implies the importance of multimessenger astronomy for the coming generations of ground based detectors.

\section{Full parameters set estimation}

In the above two subsections, we have investigated the lensing effect on binary parameters part and lens parameters part individually. But in many cases we cannot know any part of the parameters in advance. So in this subsection we will consider the four parameters $M_{l z}, y, \mathcal{M}$ and $\nu$ altogether.

First, we use the Fisher matrix method to estimate roughly the parameters extraction error. We can find out that the error estimate for the full parameters set is roughly the same as the individual binary parameters subset and lens parameters subset. The correlation matrix reduced from the Fisher matrix also shows that the correlation between these two parameter sets is weak. As an example, for the case of $\mathrm{BH}-\mathrm{BH}, M_{l z}=1000 M_{\odot}, y=0.1$ with the AdvLIGO detector, the correlation coefficient between $M_{l z}$ and $y$ is 0.964 , the coefficient between $\mathcal{M}$ and $\nu$ is -0.870 . While the correlation coefficient between $\mathcal{M}$ and $M_{l z}$ is 0.0095, the coefficient between $\mathcal{M}$ and $y$ is -0.0035 , the coefficient between $\nu$ and $M_{l z}$ is -0.0044 , and the coefficient between $\nu$ and $y$ is 0.0075 . Here the correlation coefficient is defined as $F_{i j} / \sqrt{F_{i i} F_{j j}}$ with $F_{i j}$ the element of the Fisher matrix. 

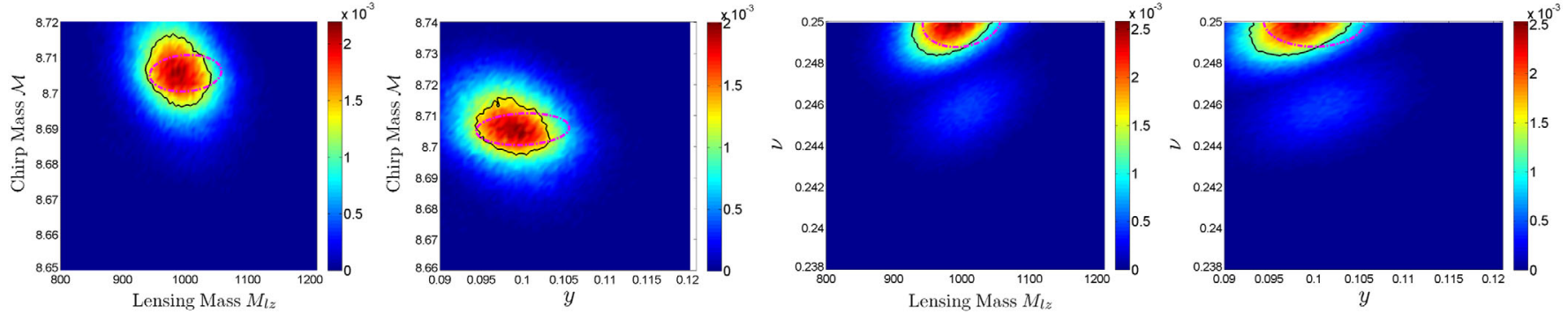

FIG. 5 (color online). Joint PDFs for parameters between the binary parameters subset $\mathcal{M}, \nu$ and the lens parameters subset $M_{l z}, y$. In these plots, $M_{l z}=1000 M_{\odot}$ and the point lensing model are used. These plots are for BH-BH sources and Advanced LIGO. The plot convention is the same as Fig. 2.

We have used MCMC simulation to confirm the above result. Based on the MCMC chain, we can calculate the correlation matrix which has elements $\Sigma_{i j} / \sqrt{\Sigma_{i i} \Sigma_{j j}}$, and $\Sigma_{i j} \equiv \frac{1}{N} \Sigma_{n=1}^{N} \theta_{i}^{(n)} \theta_{j}^{(n)}$, where $\theta_{i}^{(n)}$ is the value of the $i$ th parameter at the $n$th drop during the MCMC simulation. For the case of BH-BH, $M_{l z}=1000 M_{\odot}, y=0.1$ with the AdvLIGO detector, the correlation coefficient between $M_{l z}$ and $y$ is 0.81 , and the coefficient between $\mathcal{M}$ and $\nu$ is -0.82 . While the correlation coefficient between $\mathcal{M}$ and $M_{l z}$ is -0.19 , the coefficient between $\mathcal{M}$ and $y$ is -0.19 , the coefficient between $\nu$ and $M_{l z}$ is -0.11 , and the coefficient between $\nu$ and $y$ is -0.11 . In Fig. 5 we show the MCMC resulted joint PDFs of two parameters, one of which comes from the binary parameters subset and the another of which comes from the lensing parameters subset. In this plot we use the PN wave form model together with the point lensing model and $M_{l z}=1000 M_{\odot}$. This figure is for the $\mathrm{BH}-\mathrm{BH}$ source and AdvLIGO. For other cases, the result is similar. From these plots, all of the PDFs show a weak particular direction. This implies that the correlation between these parameters is weak. This is consistent to the finding with the above correlation coefficients. The measurement error estimate obtained in the above two subsections is roughly the same as the one gotten through full parameters MCMC simulations. The cases investigated here imply that for the lensed gravitational signal, one can treat the binary parameters and the lensing parameters individually.

\section{SUMMARY AND DISCUSSION}

General relativity describes light bending which results in gravitational lensing of optics. Such a phenomena has been widely used in astronomy and cosmology. The analogous mechanism also happens to gravitational waves. Due to the long wavelength property of gravitational wave, the lensing effect of gravitational wave is stronger than that of electromagnetical waves in a similar condition. Recent studies in the literature imply that the event rate for gravitational lensing of gravitational wave may not be ignored for the space based gravitational wave detector and for the next generations of ground based detectors.
Assuming the lensed gravitational wave signal has come into the detector, we investigated the possible loss if we use the gravitational wave form template without lensing to do the matched filtering. Currently the lensing effect has not been taken into serious consideration by the LIGO community. So using an unlensed template to analyze lensed wave signal is very possible to happen in the near future. Our results imply that this kind of treatment may result in severe loss of signal detection for the lensed gravitational wave when the lens object is massive $\left(>10^{3} M_{\odot}\right)$. In contrast, if the lensed wave form model is used for the template, useful information can be extracted via lensed gravitational wave detection. Regarding this problem, we investigated how the lensing effect may affect the parameter extraction for the binary objects sector. Our result shows lensing effect mildly affects this parameter estimation. As one expects, the lensing effect allows one to constrain the lens parameters. But this happens in a nontrivial way. If the gravitational wave detection is used alone, the parameter extraction gives large error which makes the information extraction quite hard. Instead the lensed gravitational wave detection may result in a stringent relation between the lensing mass $M_{l z}$ and lensing strength parameter $y$. So if one can combine this result with other observation results, say the mass $M_{l z}$ coming from an optical observation, the parameters of lens source can be determined accurately. Our result implies the importance of multimessenger astronomy in the coming advanced detector era.

\section{ACKNOWLEDGMENTS}

This work was supported by the NSFC (No. 11205226 and No. 11375260). The work of Y. W. was partially supported by DFG Grant No. SFB/TR 7 Gravitational Wave Astronomy and DLR (Deutsches Zentrum für Luftund Raumfahrt). Y. W. also would like to thank the German Research Foundation for funding the Cluster of Excellence QUEST-Center for Quantum Engineering and Space-Time Research. L.F. L. would like to appreciate the Specialized Research Fund for State Key Laboratories. 


\section{APPENDIX A: PN WAVE FORM MODEL}

The related quantities in the PN wave form model (A1) can be explicitly written as

$$
\begin{gathered}
\mathcal{M}=M \nu^{3 / 5}, \quad D_{\mathrm{eff}}=\frac{D}{\sqrt{F_{+}^{2}\left(1+\cos ^{2} \imath\right) / 4+F_{\times}^{2} \cos ^{2} l},} \\
F_{+}=-\frac{1}{2}\left(1+\cos ^{2} \theta\right) \cos 2 \phi \cos 2 \psi-\cos \theta \sin 2 \phi \sin 2 \psi, \\
F_{\times}=\frac{1}{2}\left(1+\cos ^{2} \theta\right) \cos 2 \phi \sin 2 \psi-\cos \theta \sin 2 \phi \cos 2 \psi, \\
\Psi(f)=2 \pi f t_{c}-\phi_{c}-\frac{\pi}{4}+\frac{3}{128 \nu v^{5}}\left[1+\frac{20}{9}\left(\frac{743}{336}+\frac{11}{4} \nu\right) v^{2}-16 \pi v^{3}+10\left(\frac{3058673}{1016064}+\frac{5429}{1008} \nu+\frac{617}{144} \nu^{2}\right) v^{4}\right. \\
+\pi\left(\frac{38645}{756}-\frac{65}{9} \nu\right)\left\{1+3 \ln \left(\frac{v}{v_{\mathrm{lso}}}\right)\right\} v^{5}+\left\{\frac{11583231236531}{4694215680}-\frac{640}{3} \pi^{2}-\frac{6848 \gamma}{21}-\frac{6848}{21} \ln (4 v)\right. \\
\left.\left.+\left(-\frac{15737765635}{3048192}+\frac{2255 \pi^{2}}{12}\right) \nu+\frac{76055}{1728} \nu^{2}-\frac{127825}{1296} \nu^{3}\right\} v^{6}+\pi\left(\frac{77096675}{254016}+\frac{378515}{1512} \nu-\frac{74045}{756} \nu^{2}\right) v^{7}\right],
\end{gathered}
$$

where $v=(\pi M f)^{1 / 3}$ and $v_{\text {lso }}=1 / \sqrt{6}$. In the above expressions, $M=m_{1}+m_{2}$ is the total mass of the binary, $D$ is the distance between the binary and the earth, $(\theta, \phi)$ is the sky location of the binary, $\psi$ is the polarization angle, and $\iota$ is the inclination angle between the rotation axis of the binary system and the direction of observation.

\section{APPENDIX B: LENSING MODELS FOR GRAVITATIONAL WAVE}

The lensing model can be described with a complex amplify function $F$. In this paper we consider the point mass lens model and the singular isothermal sphere (SIS) lens model. For the point mass lens model we have

$$
F(f)=\exp \left\{\frac{\pi w}{4}+i \frac{w}{2}\left[\ln \left(\frac{w}{2}-2 \phi_{m}^{p}\right)\right]\right\} \Gamma\left(1-\frac{i}{2} w\right){ }_{1} F_{1}\left(\frac{i}{2} w, 1 ; \frac{i}{2} w y^{2}\right)
$$

where $w=8 \pi M_{l z} f, \phi_{m}^{p}=\left(x_{m}-y\right)^{2} / 2-\ln x_{m}$ with $x_{m}=\frac{y+\sqrt{y^{2}+4}}{2}, M_{l z}$ is the effective lens mass and ${ }_{1} F_{1}(\cdot, \cdot ; \cdot)$ is the confluent hypergeometric function which is also called complex Kummer function. For the singular isothermal sphere model, we have

$$
F(f)=-i w e^{i w y^{2} / 2} \int_{0}^{\infty} x J_{0}(w x y) \exp \left\{i w\left[\frac{x^{2}}{2}-x+\phi_{m}^{s}\right]\right\}
$$

where $J_{0}$ is the Bessel function of zeroth order, $\phi_{m}^{s}=y+1 / 2$.

[1] J. K. Lawrence, Nuovo Cimento Soc. Ital. Fis. 6B, 225 (1971).

[2] H. C. Ohanian, Phys. Rev. D 8, 2734 (1973).

[3] T. T. Nakamura, Phys. Rev. Lett. 80, 1138 (1998).

[4] R. Takahashi, Astron. Astrophys. 423, 787 (2004).

[5] Y. Wang, A. Stebbins, and E. L. Turner, Phys. Rev. Lett. 77, 2875 (1996).

[6] C. Baraldo, A. Hosoya, and T. T. Nakamura, Phys. Rev. D 59, 083001 (1999).

[7] G. Bimonte, S. Capozziello, V. Manko, and G. Marmo, Phys. Rev. D 58, 104009 (1998).
[8] R. Takahashi and T. Nakamura, Astrophys. J.595, 1039(2003).

[9] A. J. Moylan, D. E. McClelland, S. M. Scott, A. C. Searle, and G. Bicknell, arXiv:0710.3140.

[10] N. Seto, Phys. Rev. D 69, 022002 (2004).

[11] K. Yamamoto and K. Tsunoda, Phys. Rev. D 68, 041302 (2003).

[12] T. Suyama, R. Takahashi, and S. Michikoshi, Phys. Rev. D 72, 043001 (2005).

[13] C.-M. Yoo, K.-i. Nakao, H. Kozaki, and R. Takahashi, Astrophys. J. 655, 691 (2007). 
[14] N. Matsunaga and K. Yamamoto, J. Cosmol. Astropart. Phys. 01 (2006) 023.

[15] M. A. Varvella, M. Angonin, and P. Tourrenc, Gen. Relativ. Gravit. 36, 983 (2004).

[16] M. Sereno, A. Sesana, A. Bleuler, P. Jetzer, M. Volonteri, and M. C. Begelman, Phys. Rev. Lett. 105, 251101 (2010).

[17] K. Danzmann and A. Rüdiger, Classical Quantum Gravity 20, S1 (2003).

[18] P. Amaro-Seoane, N. Andersson, K. Arun, S. Bose, L. Bosi, T. Bulik, J. Clark, T. Dent, J. Gair, K. Glampedakis et al., Codified Document No. ET-030-09, 2009.

[19] A. Piórkowska, M. Biesiada, and Z.-H. Zhu, J. Cosmol. Astropart. Phys. 10 (2013) 022.

[20] K. G. Arun, B. R. Iyer, B. S. Sathyaprakash, S. Sinha, and C. Van Den Broeck, Phys. Rev. D 76, 104016 (2007).

[21] N. J. Cornish and J. Crowder, Phys. Rev. D 72, 043005 (2005).

[22] A. Buonanno, B. R. Iyer, E. Ochsner, Y. Pan, and B. S. Sathyaprakash, Phys. Rev. D 80, 084043 (2009).

[23] C. Van Den Broeck, Classical Quantum Gravity 23, L51 (2006).

[24] F. Foucart, L. Buchman, M. D. Duez, M. Grudich, L. E. Kidder, I. MacDonald, A. Mroue, H. P. Pfeiffer, M. A. Scheel, and B. Szilagyi, Phys. Rev. D 88, 064017 (2013).

[25] K. G. Arun, B. R. Iyer, B. S. Sathyaprakash, and P. A. Sundararajan, Phys. Rev. D 71, 084008 (2005).
[26] M. C. Miller and D. P. Hamilton, Mon. Not. R. Astron. Soc. 330, 232 (2002).

[27] S. F. P. Zwart and S. L. McMillan, Astrophys. J. 576, 899 (2002).

[28] S. F. P. Zwart, H. Baumgardt, P. Hut, J. Makino, and S. L. McMillan, Nature (London) 428, 724 (2004).

[29] J. Gerssen, R. P. van der Marel, K. Gebhardt, P. Guhathakurta, R. C. Peterson, and C. Pryor, Astron. J. 124, 3270 (2002).

[30] K. Gebhardt, R. M. Rich, and L. C. Ho, Astrophys. J. 634, 1093 (2005).

[31] E. Noyola, K. Gebhardt, and M. Bergmann, Astrophys. J. 676, 1008 (2008).

[32] D. Shoemaker (LIGO Scientific Collaboration), https:// dcc.ligo.org/cgi-bin/DocDB/ShowDocument?docid=2974 (2010).

[33] C. K. Mishra, K. G. Arun, B. R. Iyer, and B. S. Sathyaprakash, Phys. Rev. D 82, 064010 (2010).

[34] B. Kocsis, Astrophys. J. 763, 122 (2013).

[35] T. A. Apostolatos, Phys. Rev. D 52, 605 (1995).

[36] P. Jaranowski, A. Królak, and B. F. Schutz, Phys. Rev. D 58, 063001 (1998).

[37] C. L. Rodriguez, B. Farr, W. M. Farr, and I. Mandel, Phys. Rev. D 88, 084013 (2013).

[38] C. Cutler and E. E. Flanagan, Phys. Rev. D 49, 2658 (1994).

[39] S. Nissanke, D. E. Holz, N. Dalal, S. A. Hughes, J. L. Sievers, and C. M. Hirata, arXiv:1307.2638. 